\title{
Intraoperative Incidents and Complications during Surgical Removal of Impacted and Semi-Impacted Third Molars. A Retrospective Case Study and Review of Literature
}

\section{Florou $\mathrm{E}^{\mathbf{1}}$ and Kolomvos Nikolaos ${ }^{2 *}$}

${ }^{1}$ Postgraduate Student, Department of Oral Surgery, Dental School, National and Kapodistrian University of Athens, Greece

${ }^{2}$ Assistant Professor, Department of Oral and Maxillofacial Surgery, Dental School,

National and Kapodistrian University of Athens, Greece

*Corresponding Author: Kolomvos Nikolaos, Department of Oral and Maxillofacial

Surgery, School of Dentistry, National and Kapodistrian University of Athens, Greece.
Received: June 02, 2021

Published: June 23, 2021

(C) All rights are reserved by Florou $\mathbf{E}$ and

Kolomvos Nikolaos.

\section{Abstract}

Surgical removal of third molars is a common procedure in oral surgery. Complications that may occur are interoperative bleeding, displacement of third molar, apex displacement, damage to adjacent teeth, fracture of the alveolar process, maxillary tuberosity or mandible, oroantral communication, nerve injury, tool breakage, burn of soft tissues, emphysema and injury of the temporomandibular joint. This article provides a retrospective study of specific cases with interoperative complications and a literature review. 28 cases of patients were involved in the study after the occurrence of complications during the removal of third molars of the upper and lower jaw.

Keywords: Impacted; Semi-Impacted Third Molars; Complications; Surgical Removal Per Operative Complication; Bleeding; Bone Fracture; Displacement

\section{Introduction}

Intraoperative and postoperative complications during surgical removal of third molars of the upper and lower jaw occur at rates of 4.6 to $30.9 \%$ [1-3]. Complications are usually due to poor assessment of the case, ignorance of the anatomy of the area, overestimation of the potentials of the surgeon, practice of excessive and incorrect force and also, lack of proper tools [1-3]. Proper preoperative planning, adherence to the principles of surgical techniques and complete information of patients about the complications that may arise can reduce their appearance, but also make it easier to treat them if they eventually occur [1].

Factors that affect the incidence of complications are age, gender, medical history, type of impaction, the relationship of the teeth to the inferior alveolar nerve and the lingual nerve as regards to the third molars of the lower jaw as well as their relationship with the maxillary sinus (antrum of Highmore) as regards to the third molars of the upper jaw, the technique of anesthesia, the experience of the surgeon, etc. and also anxiety and fear of the patient [1-4].

The incidents and the main intraoperative complications of surgical removal of the third molars of the upper and lower jaw in order of importance and frequency are as follows [5,6]:

- Primary bleeding

- Displacement of the third molar or part of it or teeth migration

- Oro-antral communication (regarding the third molars of the upper jaw) 
- Apex displacement in the mandibular canal or maxillary sinus

- Nerve injury (inferior alveolar nerve, lingual nerve)

- Fracture of the alveolar process, maxillary tuberosity or mandible

- Fracture of the tooth

- Damage to adjacent teeth

- Tool breakage

- Subcutaneous or submucosal emphysema

- Injury, tearing or thermal burn of soft tissues

- Injury of the temporomandibular joint or temporo-maxillary fosses.

This article provides a retrospective study of specific cases with incidents and interoperative complications during the surgical removal of third molars and a literature review.

\section{Materials and Methods}

Twenty-eight cases of patients were involved in the study after the occurrence of intraoperative complications during the removal of third molars of the upper and lower jaw (Table).

\begin{tabular}{|l|c|}
\hline \multicolumn{1}{|c|}{ Complications No } & \\
\hline Local interoperative bleeding & 8 \\
\hline Interoperative bleeding of soft tissues & 1 \\
\hline Displacement of impacted third molar & 3 \\
\hline Apex displacement & 2 \\
\hline $\begin{array}{l}\text { Split of cortical bone by dental elevator (distal of } \\
\text { second molars }\end{array}$ & 7 \\
\hline Fracture of the mandible & 1 \\
\hline Damage to adjacent teeth & 1 \\
\hline Tool breakage & 2 \\
\hline Thermal burn & 2 \\
\hline Temporomandibular joint dislocation & 1 \\
\hline
\end{tabular}

Table: Number of procedures with intraoperative incidents and complications during surgical removal of third molars of the upper and lower jaw.

\section{Results}

Eight cases of patients with primary local interoperative bleeding due to extensive injury and lack of hemostasis were reported during the removal of a third molar of the upper or lower jaw. In one case reactive bleeding and injury of the mucosa was found at the junction of soft and hard palate on the left side that occurred during the attempt to remove the third molar of the lower jaw. The elevator caused the injury due to lack of controlled leverage (Figure 1).

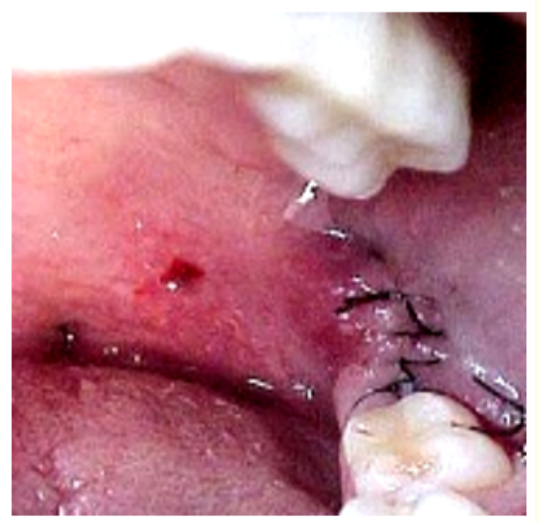

Figure 1: After reactive bleeding of the soft palate.

In three patients there was a displacement of the third molar. Specifically, in one case displacement of a third molar of the upper jaw took place within the maxillary sinus with simultaneous oroantral communication, in one case in the left infratemporal space due to excessive force and incorrect technique (Figure 2), while in one case the third molar of the lower jaw was displaced sublingually in the left submandibular and lateral pharyngeal area due to a distal-lingual position of the semi-impacted third molar and uncontrolled force during its leveraging (Figure 3).

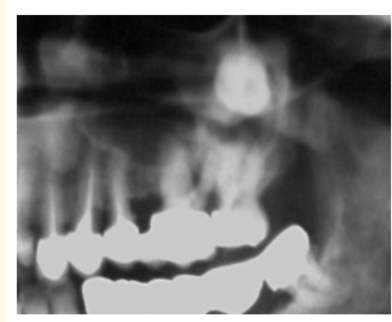

A

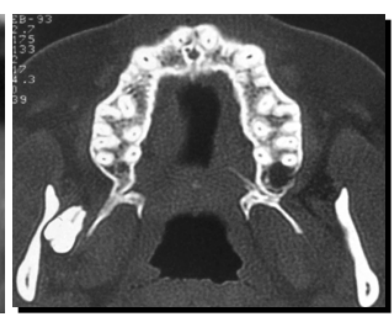

B
Figure 2: Displacement of \#28 and \#18 in the infratemporal space (A: Panoramic x-ray, B: Cone-Beam Computed Tomography). 


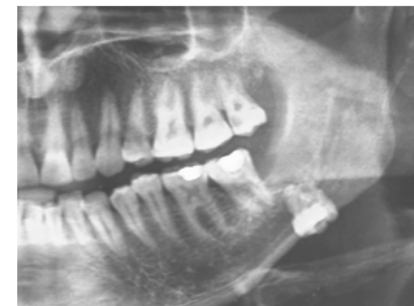

A

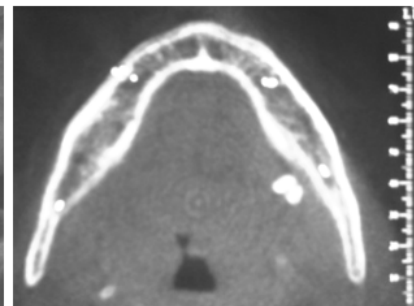

B
Figure 3: Displacement of \#38 in the submandibular space (A: Panoramic x-ray, B: Cone-Beam Computed Tomography).

In two cases, displacement of the apex of a third molar of the lower jaw in the mandibular canal occurred during the attempt to remove it with a root apex elevator. In seven cases a split of the thin cortical bone distal-lingual of the second molar of the mandible occurred due to uncontrolled use of the elevator. In one case, a fracture of the mandible occurred during the leveraging of a horizontal third molar on the right side as there was no attempt to divide the tooth before removing it (Figure 4).

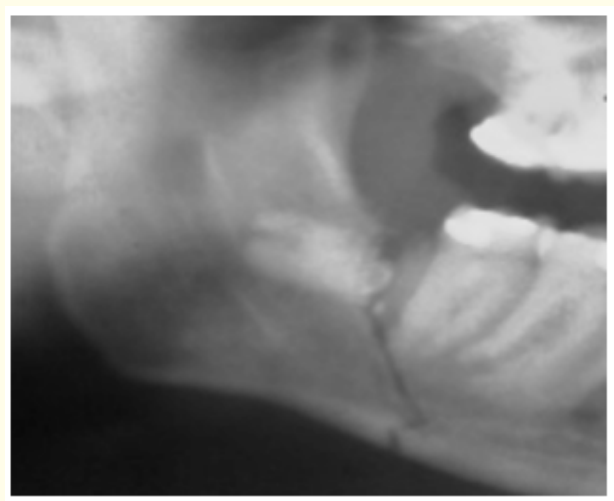

Figure 4: Fracture of the mandible during the attempt to remove the impacted \#48.

In one case, damage was caused to the adjacent tooth and more specifically a crown-root fracture of the decayed second molar during the attempt to deleverage the left third molar of the lower jaw because the tooth was used as a fulcrum and the elevator was used incorrectly, with uncontrolled and extensive force (Figure 5). This tooth was vital, because was the last one in this part of the mouth and there was the adverse tooth above this.

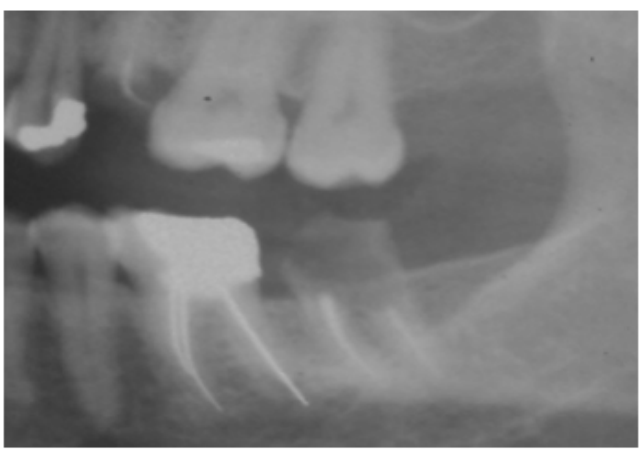

Figure 5: Crown-root fracture of the adjacent tooth (\#37) during the extraction of the third molar (\#38).

In two cases, a tool that was lodged in the around tissues broke. In one patient the elevator tip broke while attempting to remove the left third molar of the mandible (Figure 6), while in the second patient the needle of the syringe broke during the inferior alveolar nerve block (Figure 7).

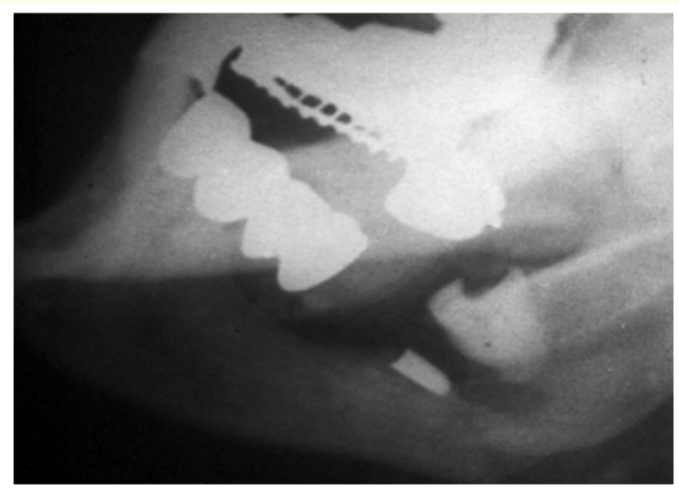

Figure 6: Breakage of an elevator during the attempt to remove the third molar (\#38).

Finally, in two cases a burn occurred from the use of an overheated angular handle (Figure 8), while in one case dislocation of 


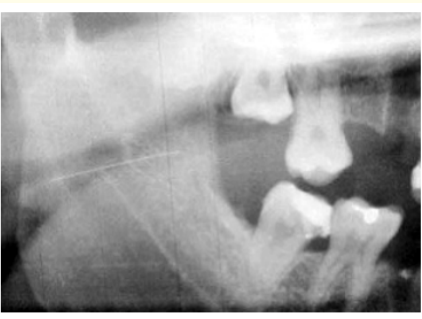

A

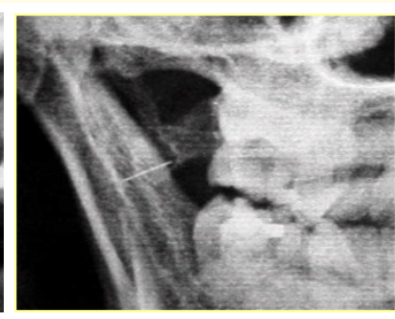

B
Figure 7: Breakage of the needle of a syringe in the pterygomandibular space during the inferior alveolar nerve block (A: Panoramic X ray, B: 3D X ray).

the temporomandibular joint on the right side occurred due to the opening of the mouth for a long time and the presence of a plane mandibular fossa of the temporal bone.

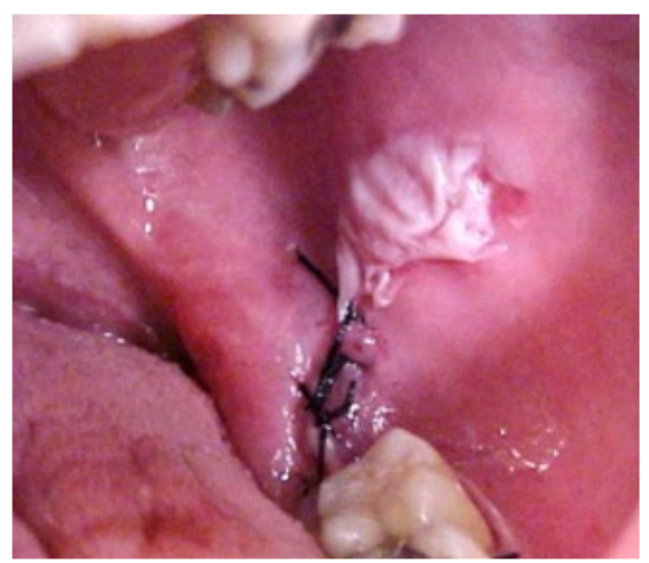

Figure 8: Burn of the buccal mucosa due to the use of overheated handle during the removal of the third molar (\#38).

\section{Discussion}

The incidents and basic intraoperative complications that occur during the procedure of removal of the third molars of the upper and lower jaw are as follows.
Bleeding: Clinically significant bleeding occurs in $0.2 \%-5.8 \%$ both intraoperatively and postoperatively and sometimes can be life-threatening for the patient [1]. The unexpected intraoperative bleeding occurs in $0.7 \%$ [4]. It is distinguished in: Expected, primary, secondary and reactive (mainly related to soft tissues).

Primary bleeding occurs during the operation and is due to extensive injury and lack of hemostasis. Reactive bleeding of the soft tissues lingual and distal from the third molar of the lower jaw occurs if the elevator is used with excessive force and without control and as a result causes the injury of the soft tissues. Generally, the causes of bleeding are divided into local and systematic. Its effect increases in teeth with distal angle, deep impaction as well as in elderly patients [7]. In addition, the frequency is higher in the removal of the third molars of the mandible than in the upper third molars (80\% and 20\% respectively) [8]. Systemic factors include mainly haemophilia A or B, von Willebrand disease and anticoagulant or antiplatelet therapy. In this case it is necessary to take local hemostatic measures and if appropriate discontinuance of antithrombotic drugs and heparinism in case of discontinuance of per os anticoagulants [8]. In addition, it may be necessary to administer the missing coagulation factors before the surgery. Massive intraoperative bleeding is rarely observed. It is likely due to some arteriovenous dysplasia usually of the lower jaw, which may be characterized of low (venous) or high (arterial) flow. These malformations are rare in the maxillofacial area and may be evident in a physical examination or in a panoramic X-ray. The most common objective point that can create suspicion is the history of repeated spontaneous bleeding from the gums. Other signs are gum discoloration, hyperthermia over damage, and subjective palpitation in the area. Angiography is required for diagnosis. Treatment is surgical removal or embolism $[1,9,10]$.

Proper design and execution of the incision and the whole operation in general, which are based on the knowledge of the adjacent anatomical elements in order to avoid their injury, reduces the chances of developing interoperative bleeding.

Displacement of third molars: It can occurs during their extraction, especially when attempted with extraction forceps and the tooth has a rhomboid crown shape and conical roots and as a result escapes from the extraction tool. The third molars of the lower jaw may be displaced sublingually, in the submandibular or lateral 
Intraoperative Incidents and Complications during Surgical Removal of Impacted and Semi-Impacted Third Molars. A Retrospective Case Study and Review of Literature

pharyngeal areas or in the pterygomandibular space [11,12]. Factors that increase the risk are the position of the tooth (distal-lingual), the thin lingual cortical plate and excessive or uncontrolled force during the use of elevators.

To avoid the displacement, the periosteal elevator is placed lingually in the retro-molar triangle and this also protects the lingual nerve at the same time. Usually when the teeth go through a perforation of the lingual plate they end up under the mylohyoid muscle. During the attempt to remove the displaced tooth in this area caution is required to avoid injury of the lingual nerve, since detection is difficult and limited visibility combined with bleeding and movements of the patient often lead to further displacement [1].

The displacement of the upper molars is a rare complication. When located deep in the mandible they may be separated by only one thin bone plate from the infratemporal space and the third molar can be displaced within this space if the distal deleverage is not accompanied by a retractor placed behind the maxillary tuberosity inside the mucoperiosteal flap. Also, a periosteal elevator must be placed behind the maxillary tuberosity. In case of displacement, the exact location of the tooth is detected after a detailed radiological examination and then it is removed [1]. Displacement of the third upper molar may also occur in the maxillary sinus due to excessive force with wrong direction and incorrect surgical technique $[11,13]$. In this case the tooth should be removed so that contamination does not occur and then the oro-antral communication is treated.

Oro-antral communication: Oro-antral communication occurs most often during the extraction of the first and second upper molars [14]. Its incidence during the extraction of third molars is $0.008-0.25 \%$, but this percentage may be underestimated as even if communication occurs it is often self-recovering $[7,15]$. Also in impacted molars a flap is advanced and sutured. As a result healing occurs. Automatic healing also occurs in communication smaller than $2 \mathrm{~mm}[16,17]$. Many techniques have been described for the closure of oro-antral communication (especially if the communication is large there is a precision technique - bichat's ball), but if communication is left untreated then it gets epithelialized and an oro-antral fistula is developed [1].

Displacement of a tooth apex in the mandibular canal: It is a relatively serious complication, because during the attempt to re- move the apex, an injury of the inferior alveolar nerve and artery may occur resulting in a different degree of hypesthesia that can remain from 6 months to years. When an apex displacement occurs, the apex is detected and removed [18].

Nerve injury (inferior alveolar nerve, lingual nerve): The injury may be accidental if for example the inferior alveolar nerve is located between the roots of a molar in which case it can be carried away and and get torn during the removal of the tooth or even during the curettage of the apical part of the socket [18].

The lingual nerve may be injured when removing third lower molars, especially when their impaction is in a distal- lingual direction $[19,20]$. Also, when bone distal from the third lower molar is removed or when a lingual flap is developed there is an increased chance of injury of the lingual nerve [19]. Hypesthesia or anesthesia are caused, lasting a few weeks up to months (6 - 24 months).

For avoidance of nerve injury, the dentist needs to insist for importance of pre-operator study and radiographic consideration, with pre-medication nerve protection.

\section{Fracture of the alveolar process, maxillary tuberosity or man-}

dible: Alveolar process fracture usually occurs when the tooth has synostosis and excessive force is applied during its extraction. If the fractured bone is completely detached from the soft tissues or periosteum then it is removed. However, if it is attached to the periosteum so that it has adequate blood supply, it remains in the area and the wound is sutured. If during extraction it is found that a large part of the alveolar process is carried away, the tooth is repositioned, immobilized and after a certain period the tooth is surgically removed [21].

Fracture of the mandible during or after removal of the third lower molar is a rare but serious complication with an incidence of approximately $0.0049 \%$ [22]. Factors that increase the risk are advanced age, mandible atrophy, deep impaction, the presence of a cyst or tumor, osteoporosis and osteomyelitis. It is usually due to the application of excessive pressure during extraction and depends on the anatomical position of the tooth and the technique followed (insufficient bone removal). The area next to the angle of the mandible shows lower resistance to fractures due to its fine dimensions and the fact that the tooth takes up space in the bone. If there is an odontogenic or dentigerous cyst around the third mo- 
Intraoperative Incidents and Complications during Surgical Removal of Impacted and Semi-Impacted Third Molars. A Retrospective Case Study and Review of Literature

lar or a peri-apical (radicular) cyst of the second molar then the mandible in this area is weaker. Fractures starting at the point of extraction are not always easily identified and if there is suspicion we do a CBCT if the fracture isn't visible in the panoramic X-ray [1].

The incidence of fractures of the maxillary tuberosity when removing third upper molars is $0.6 \%$ [7]. They are due to the application of excessive force (especially if the forces are distal). If a fracture occurs and the tooth is asymptomatic it can remain in place and become immobilized. As a precaution it is important to carefully separate the tooth from the periodontal ligament and palpate the alveolar process with the finger of the other hand in order to assess the dilatation of the cortical plate during the deleverage [1].

Crown or root fracture: It is mainly due to the practice of excessive and incorrect force, inappropriate use of tools or lack of proper tools. It occurs mainly on extensive caries and the application of excessive force.

Damage to adjacent teeth: Displacement, fracture, extraction of an adjacent tooth may occur due to improper use of tools or improper technique and especially if the adjacent tooth is used as a fulcrum. Adjacent teeth with large restorations or extensive caries present a risk of fracture or damage. The mesial and second-class impaction of the third molar carries a greater risk of damage to the second molar [7]. Full information to the patient about the possibility of this complication before the operation or if it finally occurs, is necessary. Proper use of the extraction tools and conservative bone removal around the tooth to be removed can help prevent this complication.

Tool breakage: Mainly concerns the tip of a tool (e.g. elevator) that may be lodged in the area of extraction. The fragment is detected radiologically and surgically removed simultaneously with the extraction of the tooth. It can also rarely occur breakage of the needle of the syringe during the anesthesia of the inferior alveolar nerve. This complication can have life-threatening consequences when vital anatomical structures are involved [23]. In the past the cause of the complication was breakage due to fatigue of a sterile reusable anesthesia needle due to repeated sterilization. Durable disposable stainless steel needles are now used [24]. The majority of cases are due to sudden movement of the patient during the commission of anesthesia [23,24].
Changing the direction of the needle inside the tissues can also cause it to break [25]. Cases have been reported where the broken part of the needle has been moved to the base of the skull, to the external ear canal or even near important arteries of the head and neck [26-31]. Patients may experience symptoms of pain or tenderness, trismus, swelling, dysphagia or be asymptomatic. Unilateral hearing loss has also been reported. To avoid the above, the needle should not be bent. When a change in its direction is required, it should completely exit the tissues and enter again. Also check for construction errors, choice of correct diameter, correct technique and handling of the patient are factors that should be taken into account. Long needles are considered safer because even if they break part of them protrudes from the tissues and so they can be removed more easily [23].

For the same reason, the entire needle may not enter the tissues but should remain at least $5 \mathrm{~mm}$ apart [25]. It can be detected first by panoramic X-ray and then by CT scan of conical beam (CBCT) which is considered the method of selection [23,32,33]. With CBCT also neighbouring structures, such as important vessels and the parotid gland, can be checked [34].

Subcutaneous or submucosal emphysema: Occurs if an air turbine or air syringe is used during the operation (e.g. for tooth cross-section or field cleaning by blowing). It can also occur from carrying out a Valsava test on behalf of the patient. It can also rarely occur after removal of the tooth, in which case by increasing the intra-oral pressure of the air (e.g. blowing with closed mouth) air can pass into the loose connective tissue from the detached mucosa and become trapped there. A characteristic diagnostic point, in addition to the swelling, is the sound and sensation (crepitus) during palpation. The prognosis is good because it passes progressively in 2 - 4 days, while if the emphysema is extensive and concentrated some air can be removed by aspiration. To avoid this, the use of airotor (for cutting tooth or bone) is prohibited. Note that in addition to local emphysema, trapped air can enter deep cervical areas, transferring microorganisms from the oral cavity increasing the likelihood of an infection occurring in the corresponding area or causing compression of adjacent structures. Prophylactic antibiotics may be prescribed $[35,36]$.

Injury, section, burn of soft tissues: Burn may result from the use of an overheated straight or angular handle in case the bone 
needs to be removed or the tooth cut off. Also, the elevators may injure the soft tissues if they escape control during the deleveraging attempt [2].

\section{Injury of temporomandibular joint - condylar head dislocation} It occurs from excessive opening of the mouth for a long time, as well as from excessive force on the mandible and lack of support of it when removing third molars of the lower jaw [1,37].

\section{Conclusion}

Complications happen usually due to poor assessment of the case, lack of knowledge of the anatomy of the area, overestimation of the potential of the surgeon, practice of excessive and incorrect force and lack of appropriate tools [2]. Taking a medical history and properly studying the case, preoperative radiographic evaluation and proper planning, adherence to the basic principles of surgical techniques and fully informing patients about the complications that may arise can reduce their appearance, but also make it easier to treat them if they eventually occur $[2,3]$.

\section{Bibliography}

1. Bouloux GF., et al. "Complications of third molar surgery". Oral and Maxillofacial Surgery Clinics of North America 19 (2007): 117-128.

2. Bui CH., et al. "Types, frequencies, and risk factors for complications after third molar extraction". Journal of Oral and Maxillofacial Surgery 61 (2003): 1379-1389.

3. Sisk AL., et al. "Complications following removal of impacted third molars: the role of the experience of the surgeon". Journal of Oral and Maxillofacial Surgery 44 (1986): 855.

4. Haug RH., et al. "The American Association of Oral and Maxillofacial Surgeons Age-related third molar study". Journal of Oral and Maxillofacial Surgery 63 (2005): 1106.

5. Robert D Marciani. "Complications of third molar surgery and their management". Atlas of the Oral and Maxillofacial Surgery Clinics of North America 20.2 (2012): 233-251.

6. Nabeel Sayed., et al. "Complications of Third Molar Extraction. A retrospective study from a tertiary healthcare centre in Oman". Sultan Qaboos University Medical Journal 19.3 (2019): e230-e235.
7. Chiapasco M., et al. "Side effects and complications associated with third molar surgery". Oral Surgery, Oral Medicine, Oral Pathology, and Oral Radiology 76 (1993): 412.

8. Jensen S. "Hemorrhage after oral surgery: an analysis of 103 cases". Oral Surgery, Oral Medicine, Oral Pathology, and Oral Radiology 37 (1974): 2.

9. Larsen PE and Peterson LJ. "A systematic approach to management of high-flow vascular malformations of the mandible". Journal of Oral and Maxillofacial Surgery 51 (1993): 62.

10. Kawano K., et al. "Mandibular arteriovenous malformation treated by transvenous coil embolization: a long-term followup with special reference to bone regeneration". Journal of Oral and Maxillofacial Surgery 59 (2001): 326.

11. Yeh C-J. "A simple retrieval technique for accidentally displaced mandibular third molars". Journal of Oral and Maxillofacial Surgery 60 (2002): 836.

12. Ertas U., et al. "Accidental third molar displacement into the lateral pharyngeal space". Journal of Oral and Maxillofacial Surgery 60 (2002): 1217.

13. Sverzut CE., et al. "Accidental displacement of impacted maxillary third molar: a case report". Brazilian Dental Journal 16 (2005): 167.

14. Ehrl PA. "Oroantral communication: epicritical study of 175 patients, with special concern to secondary operative closure". International Journal of Oral and Maxillofacial Surgery (1980): 351.

15. Punwutikorn J., et al. "Clinically significant oroantral communications: a study of incidence and site". International Journal of Oral and Maxillofacial Surgery 23 (1994): 19.

16. Schuchardt K. "Treatment of oro-antral perforations and fistulae". International Dental Journal (1955): 5.

17. Hanazawa Y., et al. "Closure of oroantral communications using a pedicled buccal fat pad graft". Journal of Oral and Maxillofacial Surgery 53 (1995): 771.

18. Gintaras Juodzbalys., et al. "Injury of the Inferior Alveolar Nerve during Implant Placement: a Literature". Journal of Oral and Maxillofacial Research 2.1 (2011): e1. 
19. Tojyo., et al. "Risk of lingual nerve injuries in removal of mandibular third molars: a retrospective case-control study". Maxillofacial Plastic and Reconstructive Surgery 41 (2019): 40.

20. Valmaseda-Castellón E., et al. "Lingual nerve damage after third lower molar surgical extraction". Oral Surgery, Oral Medicine, Oral Pathology, and Oral Radiology 90 (2000): 567-573.

21. Van der Weijden F., et al. "Alveolar bone dimensional changes of post-extraction sockets in humans: a systematic review". Journal of Clinical Periodontology 36 (2009): 1048-1058.

22. Libersa P., et al. "Immediate and late mandibular fractures after third molar removal". Journal of Oral and Maxillofacial Surgery 60 (2002): 163.

23. Stephan Acham., et al. "Needle fracture as a complication of dental local anesthesia: recommendations for prevention and a comprehensive treatment algorithm based on literature from the past four decades". Clinical Oral Investigations 23.3 (2019): 1109-1119.

24. Pogrel MA. "Broken local anesthetic needles: a case series of 16 patients, with recommendations". Journal of the American Dental Association 140 (2009): 1517-1522.

25. Dojcinovic I., et al. "Needle breakage: a rare and potentially dangerous complication during local anesthesia". Revue de Stomatologie, de Chirurgie Maxillo-faciale 108 (2007): 222-224.

26. Prado FB., et al. "Dental broken needle migration to the skull base, Anatomical considerations and prevention". Journal of Morphological Sciences 27 (2010): 98-101.

27. Ribeiro L., et al. "Needle in the external auditory canal: an unusual complication of inferior alveolar nerve block". Oral Surgery, Oral Medicine, Oral Pathology, and Oral Radiology 117 (2014): e436-e437.

28. Queiroz SB., et al. "Retrieval of a Broken Dental Needle Close to the Facial Artery After Cervical Migration". The Journal of Craniofacial Surgery 27 (2016): e338-e340.

29. Brooks J and Murphy MT. "A novel case of a broken dental anesthetic needle transecting the right internal carotid artery". Journal of the American Dental Association 147 (2016): 739742.
30. Casey JT., et al. "Retained dental needle migration across the skull base to the cochlea presenting as hearing loss". Otology and Neurotology 36 (2015): e42-e45.

31. Okumura Y., et al. "Unique migration of a dental needle into the parapharyngeal space: successful removal by an intraoral approach and simulation for tracking visibility in X-ray fluoroscopy". Annals of Otology, Rhinology, and Laryngology 124 (2015): 162-167.

32. Kim JH and Moon SY. "Removal of a broken needle using three dimensional computed tomography: a case report". Journal of the Korean Association of Oral and Maxillofacial Surgeons 39 (2013): 251-253.

33. Stein KM. "Use of Intraoperative Navigation for Minimally Invasive Retrieval of a Broken Dental Needle". Journal of Oral and Maxillofacial Surgery 73 (2015): 1911-1916.

34. Zeltser R., et al. "The implications of a broken needle in the pterygomandibular space: clinical guidelines for prevention and retrieval". International Journal of Paediatric Dentistry 24 (2002): 153-156.

35. YBE Tay and WS Loh. "Extensive subcutaneous emphysema, pneumomediastinum, and pneumorrhachis following third molar surgery". International Journal of Oral and Maxillofacial Surgery 47 (2018): 1609-1612.

36. McKenzie WS and Rosenberg M. "Iatrogenic subcutaneous emphysema of dental and surgical origin: a literature review". Journal of Oral and Maxillofacial Surgery 67 (2009): 12651268.

37. Huang GJ., et al. "Risk factors for diagnostic subgroups of painful temporomandibular disorders (TMD)". Journal of Dental Research 81 (2002): 284.

\section{Volume 5 Issue 7 July 2021 \\ (C) All rights are reserved by Florou E and Kolomvos Nikolaos.}

